\title{
Erratum to: Measuring, Tracking, Monitoring, and Reporting Sustainability and Resilience Progress
}

\section{Erratum to:}

Chapter 4 in: H. Alibašić, Sustainability and Resilience Planning for Local Governments, Sustainable Development Goals Series, https://doi.org/10.1007/978-3-319-72568-0_4

The original version of this chapter was inadvertently published with the incorrect spelling of 'Mentoring'. This has been corrected and the spelling has been updated as follows: "monitoring" 